\title{
Main provisions for formation of cognitive model of visually observable environment synthesized for aircraft simulator
}

\author{
Vladimir Roganov, Professor \\ Department of Information Technologies and Systems \\ Penza State Technological University, \\ Department of Information Technologies \\ Penza State University of Architecture and Construction, \\ Penza city, Russia, \\ 440000, Plekhanov street, 12-80 \\ vladimir_roganov@mail.ru \\ Ellvira Roganova, Deputy Director \\ Limited Liability Company "Video3" \\ Penza city, Russia, \\ 440008, Kulakova street, 1, office 419 \\ roganelka@mail.ru
}

\author{
Michail Miheev, Professor \\ Department of Information Technologies and Systems \\ Penza State Technological University \\ Penza city, Russia, \\ 440039, travel Baydukova / st. Gagarin, d. 1a / 11 \\ mix1959@gmail.com
}

Bolat I. Nurgozhin, Assistant professor

Department of Information Technologies

Almaty University of Power Engineering and

Telecommunications

Almaty city, Kazakhstan

e-bin15auec@gmail.ru

\author{
Valeriy Fillipenko, Assistant \\ Department of Information Technologies and Systems \\ Penza State Technological University \\ Penza city, Russia, \\ 440039, travel Baydukova / st. Gagarin, d. 1a / 11 \\ fvo304@yandex.ru
}

\begin{abstract}
Today, aviation simulators are complexes for training pilots flying an aircraft in normal situations and in preemergency situations. The pilot, piloting an aircraft simulator, manages an airplane model. The airplane model moves in a three-dimensional space model. The pilot monitors the movement of the airplane model, using information from two groups of imitators. Imitators of the first group are models of cabin equipment. The pilot receives information from them about the speed and altitude of the flight. Imitators of the second group synthesize for the pilot the image of the model of the terrain, visible through the cabin glass and on the radar and thermal imager screens. As a result, the imitators of the second group form a cognitive model of the visually observable environment, which allows the pilot to acquire professional skills in controlling the airplane during takeoff and landing on the runway and solve navigation problems while flying along the route. The article is devoted to some aspects of the formation of the cognitive model of the visually observable environment synthesized by imitators of an aircraft simulator.
\end{abstract}

Keywords - aircraft simulators; a cognitive model of a visually observable environment.; a visual situation; a radar simulator; a imitator of the thermal imager

\section{INTRODUCTION}

Aircraft simulators today are the most high-quality training systems. Initially, aviation simulators were developed to teach pilots how to fly an aircraft in normal situations and in pre- emergency situations, the appearance of which in flight can end in a catastrophe. The expansion of the list of training situations was hampered by the insufficient development of software and technical systems, as well as inadequate studies of individual imitators of the airplane simulator. Previously, in the aviation simulatoro there was only a visual imitator simulating a three-dimensional visual image of a part of the model of space visible through the glass of the cabin for the pilot. The pilot could see the three-dimensional model of the outer space only when training take-offs on the runway. The size of the visualization area with the centre of the runway was no more than $15 \times 15 \mathrm{~km}$. This part of the virtual space, in which the model of the airplane could move, was developed very well, and the pilot could navigate and solve navigation problems on the reference models of objects near the runway. When flying on an aircraft simulator "in the clouds", the pilot could navigate in a virtual space with measures of $1500 \times 1500 \mathrm{~km}$, considering a simplified image of the terrain model on the radar simulator screen. The ability to determine the location of an airplane model in a virtual space by considering the radar image of a terrain model is limited. The development of science and technology makes it possible to combine the visualization area and virtual space. For this, it is necessary to select models of reference objects and arrange them throughout the large visualization stage so that the pilot could determine the location of the airplane model over the models of reference objects. In addition, it became possible to 
use a thermal imager to solve navigation problems. The criterion for successful development of a visual simulator, a radar simulator and an imager of a thermal imager is the ability to form a cognitive model of a visually observable environment that allows the pilot to acquire professional skills in controlling an airplane and to solve navigational tasks. This article discusses this group of simulators and their main nodes.

\section{RESULTS AND DISCUSSION}

The process of development of an aircraft simulator is the selection of optical-software-technical units, called imitators, into a single complex [1]. The complex in Fig. 1 [2] is used for training pilots in specific training situations in accordance with the TOR on an aircraft simulator [3]. Previously, an aircraft simulator was designed to teach pilots the process of piloting an aircraft in normal and the pre-emergency situations [4]. This made it possible to achieve great success in the field of improving flight safety [5]. All pilots are trained of takeoff and landing on the runway using an aircraft simulator [6]. And they are trained to manage the process of elimination of known pre-emergency situations, during the flight using an aircraft simulator, too [7].

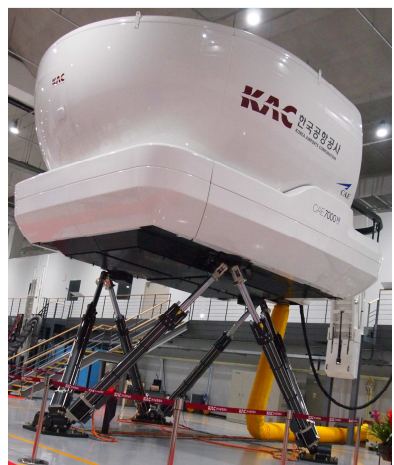

Fig.1 Comprehensive aviation simulator developed by SAE with wide-angle display device WIDE and six-degree mobility system.

However, in flight, the pilot solves the problem of piloting the aircraft and solves navigation problems, too. Today, due to a lack of known theoretical research in the field of modeling the flight process on an aircraft simulator with visibility of the earth from one turning point of the route to another, the training of pilots to solve navigational tasks of navigational tasks is limited [8]. This is a solution to navigational tasks when learning to land on a visible runway. The successes of the scientific and technological revolution, the development of BigData technology, the appearance of new materials have created the conditions for the development of new an aircraft simulator by adding situations for training pilots to solve navigational tasks.

Studies have shown that the solution of navigational tasks depends on the simulators responsible for the formation of the cognitive model of the visually observable environment. Using the cognitive model of a visually observable environment, the pilot receives $90 \%$ of the necessary information.

In the works devoted to the analysis of methods of training a pilot on an aircraft simulator, it is shown that by combining information coming from the number of imitators for the pilot, during flights on AT, the pilot achieves the feeling that he is flying on a real aircraft. Training on an aircraft simulator is a flight to an aircraft simulator. Learning to solve navigational problems on an airplane simulator is the training of a pilot for the process of orientation in space. The pilot is guided in the space, when he receives information from the imitators of cab equipment. The pilot visually determines the position of the airplane model in the space model, using a visual impersonator, radar imitator, imager imitator. Flight on an aircraft simulator is the movement of the aircraft model, in the model of external space.

Formation of the cognitive model of the visually observable environment is carried out in the real time mode due to the synthesis for the pilot of all the necessary information coming to him through the visual analyzer, with a cycle of exchange and processing of information of no more than $120 \mu \mathrm{s}$. In this process, basically three imitators are involved:

- simulator of visual situation, which is a cyber physical optical-hardware-technical system of an aircraft simulator, simulating the pilot's feeling that behind the glazing of the cabin he sees a three-dimensional image of the model of the surrounding space (Fig. 2) [8] with a quality sufficient for determining the distance to visible objects;

- radar simulator is an optical-hardware-technical aircraft simulator system that synthesizes a radar image of the terrain model section on the radar simulator screen (Fig. 3) [9], with a quality sufficient for the pilot to solve the problems of locating the aircraft model in the space model;

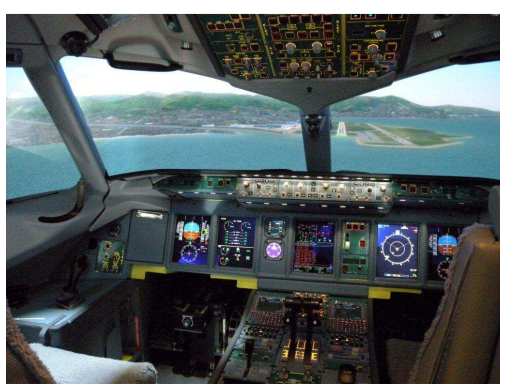

Fig.2. View of a three-dimensional model of a part of the visualization scene from the cockpit of an airplane simulator equipped with a WIDE indication device.

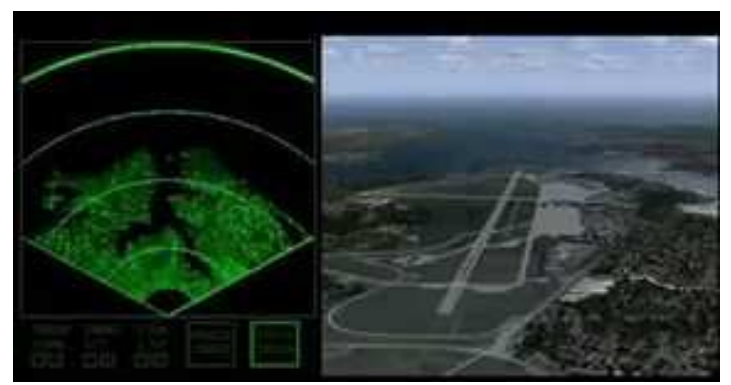

Fig.3. View from the cockpit of the aircraft to the aerodrome, and the view of the same place on the earth's surface on the radar screen in the mode of operation of the radar antenna " $120^{\circ}$ forward". 
- imitator of the thermal imager is an optical-hardwaretechnical system an aircraft simulator, synthesizing the thermal image of the terrain model section that has fallen into the pyramid of visibility on the screen of the imager of the thermal imager "Fig. 4" [10], with a quality sufficient for training the pilot to solve the problem of determining the location of the model an aircraft in the space model;

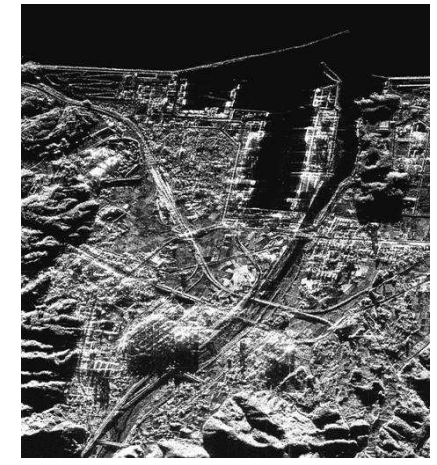

Fig.4. View of the seaside city on the thermal imager screen.

When flying on an aircraft simulator, the pilot receives information to determine the location of the model of the aircraft in the space model from the simulators of the cabin equipment, too.

This is:

1) Simulators of cabin equipment being hardwaretechnical systems of an aircraft simulator, synthesizing information on the operation of the main units of the aircraft model, the speed of its movement and the position in the space model, obtained both from models of some aircraft units (models gyroscopes, sensors, magnetic compass), as well as from the models of radio engineering means of airplane navigation.

2) The simulator of mobility is a hydro-hardware-technical aircraft simulator system. Now all complex aviation simulators are certified according to the Level D requirements for civilian simulators, which presupposes the existence of six power mobility systems for modeling accelerating sensations when changing the direction and position of the airplane model. It is believed that a person quickly catches a change in body position and, accordingly, can more quickly take measures to control the aircraft than visually processing the change in the image seen through the glazing of the cabin or by processing the indications of cabin equipment. As a result, the pilot, due to acceleration sensations, makes decisions on correcting the flight of the aircraft faster than he can visually notice the change in the position of the aircraft model in the virtual space.

An aircraft simulator can fly in a virtual space "in the clouds" or over a model of a recognizable site of the terrain. In the future, the authors will use the terms:

- "virtual space" - a three-dimensional model of a large area where an airplane model can fly (a pilot can determine the location of an airplane model in a virtual space with the help of radio engineering means of air navigation, usually during a "flight in the clouds" without land visibility;
- "visualization area" is a model of a recognizable part of the terrain over which an airplane model can fly, to teach a pilot how to pilot an aircraft and solve navigation problems ("virtual space" includes one or more "visualization areas") when the pilot flies over the "visualization area", he can determine the location of the airplane model in the virtual space using visually observable models of reference objects and with the help of radio navigation aids;

- flight pilot training is the training in professional aircraft management skills in simple and difficult weather conditions, with fail-safe operation of the main aircraft nodes, or with the failure of one or several nodes, but with the possibility of controlling the aircraft;

- learning to solve navigational problems is the training of a pilot to determine the location of an airplane in space using radio navigation aids (airborne radio compass, near-navigation radio, radar, thermal imager) when flying in clouds, or observing through the glass cabin airplane the reference objects on the ground: a river, an edge Seaside, etc., and also using the method of "reckoning the way" when the pilot determines the location of the aircraft, putting off on the map the path traversed by the aircraft from a known place.

In the "virtual space" there are mathematical models of terrestrial radio technical means of navigation and "visualization areas" with airport models. All models of virtual space are described in the world coordinate system, located in its center. The flight of the airplane model beyond the limits of the virtual space is inadmissible. Taking into account the peculiarities of the software and technical systems used, on the basis of which imitators participating in the formation of the cognitive model of the visually observable environment have been developed, rounding errors appear when moving away from the center of the world coordinate system.

When carrying out the Russian-Canadian project to develop a joint aviation simulator, while performing system analysis and collecting data on two airports in Amsterdam and Rotterdam (when landing at an airport runway in the distance, the runway of another airport is visible), it was decided to design two scenes Visualization. One scene had its center of the world coordinate system in the middle of the middle of the main runway of the Amsterdam airport. The second scene had its center of the world coordinate system in the center of the middle of the main runway of Rotterdam Airport. Two databases were developed. If the pilot is learning to land on the runway of the Amsterdam airfield, the Amsterdam airport database was chosen. If the pilot is learning to land on the runway of the Rotterdam airfield, the Rotterdam airport database was chosen. This allowed to avoid rounding errors when the pilot is acquires professional skills in landing training on a runway.

Visually observed models of objects, three-dimensional, visible through the glass of the aircraft simulator cabin, or their two-dimensional projections visible on the screens of a radar simulator or thermal imager, are synthesized in every real-time clock cycle. For this, algorithms for the synthesis of computer graphics are used. To synthesize images using computer graphics techniques, computer image generators are used - special software and hardware systems. Their purpose 
is to synthesize a two-dimensional projection of a part of a two-dimensional visualization scene. To do this, use the original information taken from pre-compiled databases, and the current view position of the observer. As a result, the corresponding image appears on the screen of the intermediate image formation. Two-dimensional projection is part of the "visualization area", which is organized by the walls of the surveillance camera. It is enough for simulators of a radar and a thermal imager. In the visual simulator, an optical display device is inserted between the pilot and the intermediate image forming screen. The purpose of the display device is to influence certain components of the human visual apparatus. Currently, there are two types of aviation simulators that use display devices to ensure that the pilot sees a threedimensional image, and aviation simulators where a complex runway landing process must be performed by instruments, only an image that helps align the aircraft is required.

If the resulting image should help the pilot to determine the distance to the observed models of three-dimensional objects, then two types of indication devices are used.

This display device uses the disparity component of human vision, the most powerful influence on the ability to see a human three-dimensional image. When used, two images are formed, one for the left eye, one for the right eye "Fig. 5". When forming such images, the center of each surveillance camera should be in place of the corresponding eye of the pilot. Such display devices, as a rule, are used for training pilots of military aircraft. They fly in headsets and, as a rule, their heads place in cabin calculate in advance where the eyes of the pilot should be in the space of the cabin of the airplane simulator. As shown by the conducted studies, the use of such display devices implies the need for a significant number of pilots (at least 47\%, when using first-standard interlaced television screens) to undergo a training course to see a threedimensional image. All people in these glasses see a clear image, but not everyone can determine the distance to the visible models of objects. However, after the training course to determine the required distance, they can do it. The maximum interval of training time, when working with such system at least 30 minutes each working day, took six months. During training, a person also has bad side effects: headache, shortterm loss of orientation in space. The rest of people immediately begin to see a three-dimensional image and evaluate the distance to the visible models of objects.

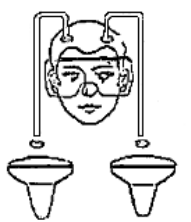

Fig.5. One of the possible schemes of a 3D system with disparant screens.

The second system of three-dimensional indication is a system based on a collimator. In this case, they affect the convergence and accommodation of human sight. Between the screen of formation of the intermediate image and the eye of the pilot, a mirror collimator is placed. Then the image "Fig. 6" falls into the eye of the person and the lens of the eye is focused on the "gaze to infinity". An obligatory condition for the use of this system is a mobile observer, or the presence of a mobile object in the field of view. And also the frequency of changing the next frame from the image in question should be above $7 \mathrm{~Hz}$. This allows one to force the accommodation and convergence of the human visual apparatus to make the pilot consider that he sees a three-dimensional image. Thus, if in the first case the visual apparatus allowed a person to see a three-dimensional image in two projections, in the latter case the visual apparatus processes the video sequence. Such system is without stereo glasses.

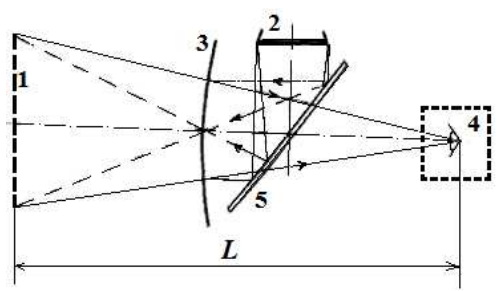

Fig. 6. Scheme of a narrow-angle 3D indicator based on a mirror collimator: 1- the place where an imaginary image appears;

2 - the screen for formation of an intermediate image;

3 - the concave spherical mirror;

4 - the pupil of observation (where, when the eye is positioned, the observer sees a three-dimensional image);

5 - beam splitter plate;

$\mathrm{L}$ - the minimum distance from the appearance of the nearest threedimensional model.

Usually such 3D indication system allows us to consider a three-dimensional model in the viewing angles $40^{\circ}$ horizontally and $30^{\circ}$ vertically. If necessary, increase the number of $3 \mathrm{D}$ indication systems using a combination of simple indicators. For example, one of the aviation simulators of the PKBM development has a three-channel display device with a total viewing angle of $120^{\circ}$ horizontally and $30^{\circ}$ vertically "Fig. 7 [12]".

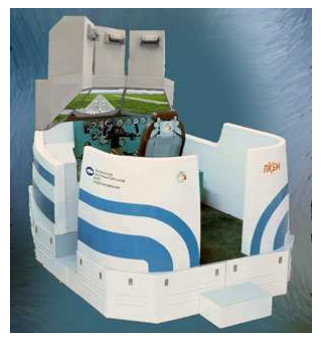

Fig. 7. Aircraft simulator with a three-channel display device with a "narrow pupil", designed for a single pilot

The system under consideration is "narrow-angle". A three-dimensional image is seen comfortably only by one person, whose eye or eyes are placed in area 4 , where the observer sees a three-dimensional image without distortion (Fig. 5). A variation "wide- angle" is the WIDE system, widely used by CAE (Canada) "Fig. 8". When using WIDE with a "wide pupil", all crew members of a wide-body aircraft can see a three-dimensional image (but with the eyes of an airplane commander) "Fig. 2".

Thus, in order to force a person to see the 3D model of space through the cabin glass of an airplane simulator, it is necessary: 
1) to influence the features of the human visual apparatus;

2) in a number of cases, to train the visual apparatus of a particular person to see a three-dimensional image of the model of the object.

This allows us to conclude that the three-dimensional display devices used in aviation simulators are pseudovolumetric, which is confirmed by the results of studies carried out by the authors.

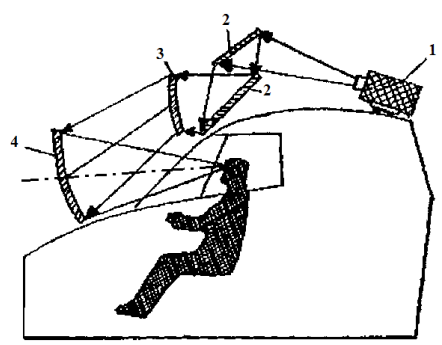

Fig.8 - Diagram of a 3D-free 3D indicator with a "wide" pupil of observation for a group of observers located in the cockpit of a cargo plane: 1 -TV projector;

2-intermediate mirrors;

3-spherical screen;

4-concave spherical mirror of large radius.

For the imitator of the radar and the imitator of the thermal imager, it is sufficient to output the colorized image accordingly on the screen of formation of the intermediate image (Fig. 3, 4).

Earlier, to obtain a three-dimensional image model, sufficient for training the pilots to visual landing on the runway model, these two indicator systems were necessarily used. This was due to the characteristics of the earlier screens used to form the intermediate image. In particular, such screens had an interlaced scan and a resolution of $640 \times 480$ pixels. The calculation of such indicator systems devices showed that the resolution of such three-dimensional display devices was 10', while the resolution of the eye of the average person is 30". This meant that some models of objects "jumped" out of the void, which did not improve the quality of training pilots.

The appearance of new television screens with progressive scanning and with better resolution, as well as the appearance on aircraft of devices that help the pilot to land on the runway, reduce the requirement for an imitator of visual situation. In many cases, it is required, when boarding a pilot, to simply see the horizon line and the location of the runway. In such cases, a widescreen screen for forming the intermediate image is used "Fig. 9" [13].

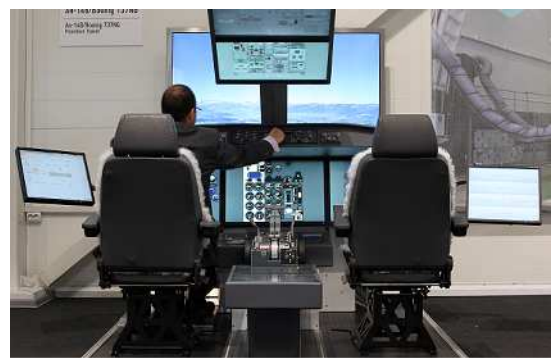

Fig.9. Aircraft simulator with a widescreen display device.
The use of only three-dimensional display devices does not allow the formation of a cognitive model of a visually observable environment. Studies have shown that it is still necessary to synthesize on the screen of formation of an intermediate image, sufficiently saturated with distinguishable models of objects. This problem is solved by two methods:

- choosing a computer image generator, the performance of which allows one to get the required set of visible models;

- using BigData technologies to obtain a sufficiently rich image, at each point of time, by creating a special database structure of the computer image generator database.

The database file structure used simulates the partitioning of virtual space into segments, with the purpose of excluding part of them that describe the invisible portion of the visualization area.

In practice, both methods are used. The performance of known computer image generators can vary from 40 visible polygons (the lights are imitated as small faces) to 300,000 visible polygons and 64,000 lights (Table 1). Currently, due to the fall in the cost of software and hardware components of computer image generators and the widespread introduction of textures (including moving textures - segments of video films), it becomes possible to select the necessary equipment depending on the tasks assigned. Choosing a computer generator is the first part of the task of developing imitators that form a cognitive model of a visually observable environment. The second stage is the solution of the problem of dividing virtual space into segments, with the goal of excluding of image synthesis at the first stage - the most part of the model of space that is not currently used. Otherwise, for a minimum size of a virtual space of $400 \times 400 \mathrm{~km}$, one cannot find a computer generator that allows one to solve only navigational learning tasks, but also the traditional tasks of training aircraft for piloting. The use of segments allows one, in the first stage, to exclude part of the virtual scene and the visualization scene from further consideration. Despite the fact that the processing time of each segment corresponds to the processing time of the polygon, the calculations show that the division into segments allows a several-time increase of the number of polygons and lights describing the selected terrain models.

TABL.1 The advertising performance of some computer generators (* noted the possibility of using a texture)

\begin{tabular}{|c|c|c|c|}
\hline $\begin{array}{l}\text { GENERATOR } \\
\text { FIRM } \\
\text { MANUFACTURER }\end{array}$ & $\begin{array}{l}\text { NUMBER OF } \\
\text { POLYGONS } \\
\text { PER CYCLE }\end{array}$ & $\begin{array}{l}\text { TIME OF } \\
\text { THE CYCLE } \\
(\mu S)\end{array}$ & $\begin{array}{l}\text { NUMBER OF } \\
\text { LIGHTS FOR } \\
\text { THE CYCLE }\end{array}$ \\
\hline $\begin{array}{l}\text { Al'batros/NPP «Era» } \\
\text { (city Penza, Russia) }\end{array}$ & 16000 & 80 & 32000 \\
\hline $\begin{array}{l}\text { Sumerki /NPP «Era» } \\
\text { (city Penza, Russia) }\end{array}$ & 10 & 120 & 32000 \\
\hline $\begin{array}{l}\text { Generator/“Video3” } \\
\text { LLC } \\
\text { (city Penza, Russia) }\end{array}$ & $40 *$ & $50 \mathrm{~Hz}$ & $\begin{array}{l}\text { The lights are } \\
\text { realized in the } \\
\text { form of small } \\
\text { polygons }\end{array}$ \\
\hline $\begin{array}{l}\text { MAXVUE }{ }^{\mathrm{TM}} / \mathrm{CAE} \\
\text { (city of Montreal, } \\
\text { Canada) }\end{array}$ & $\begin{array}{c}4000 * \text { (visible } \\
\text { polygons) }\end{array}$ & 80 & +8000 lights \\
\hline $\begin{array}{l}\text { POLIGON }{ }^{\mathrm{TM}} / \mathrm{CAE} \\
\text { (city of Montreal, ) }\end{array}$ & $300000 *$ & 80 & +64000 lights \\
\hline
\end{tabular}


Today every company that develops airplane simulators solves the problem of dividing space into segments in different ways. The most famous Canadian company SAE, which for a long time was the world leader of an airplane simulator making, divides all virtual space into segments in the form of rectangular triangles with a multiple of $625 \mathrm{~m}$ "Fig.10" with nesting levels - four.

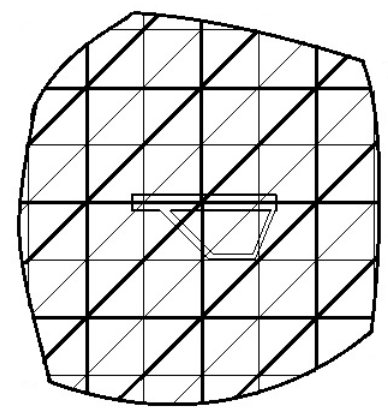

Fig.10. The virtual division is biased in segments as rectangles (two levels of nesting)

According to CAE, in the center of a virtual space of $1500 \times 1500 \mathrm{~km}$, or $1000 \times 1000 \mathrm{~km}$, there is one visualization area of $15 \times 15 \mathrm{~km}$ or $10 \times 10 \mathrm{~km}$. At the first stage of image synthesis, segments that are not caught in the footprint of the surveillance camera on the underlying surface are excluded. To accelerate the process, first segments of the first level are examined, and then smaller segments of the second level are removed from the selected segments and do not fall into the trail of the surveillance camera. Then, the third level segments are deleted. Then, segments of the fourth level are deleted. The remaining segments are obtained by primitives from whom a three-dimensional image of the required area is being recruited.

The use of BigData technologies allows one to combine the visualization scene with virtual space. In practice, this requires the joint development of three identical visualization scenes: for a visual imitator, for an imitator of a radar and for an imitator of a thermal imager. This is possible, but in the beginning, it is necessary to calculate the list of objects which models should be placed in the created scene and split the entire scene into segments so that the selected computer image generator can synthesize its image for each imitator. Experiments conducted by authors showed that this is possible. As a result, new aviation simulators can train pilots not only by piloting an aircraft, but also to teach the solution of navigation problems when flying from one turning point of the route to another from the orientation of the model of reference objects. The pilot can see three-dimensional reference objects through the cockpit glass, their twodimensional projections, but otherwise colored, on the screen of the imager of the thermal imager, and on the radar simulator screen, the pilot can see models of reference objects visible in the radio range.

\section{CONCLUSION}

Thus, as a result of the analysis, taking into account the capabilities of software and hardware, the use of new materials and research in the field of theoretical bases of information, ways of solving the problems of training pilots in aviation simulators associated with the formation of a cognitive model of a visually observable environment are determined and identified. This allowed one to create new aviation simulators and to modernize existing ones in the following directions. The first is training the piloting of an aircraft in normal and pre-emergency situations. The second is learning to solve navigational problems when landing on a runway. The third is learning to solve navigational problems when flying on air simulators from one turning point of the route to another with finding the place of the model of the aircraft in virtual space by models of reference 3D objects, located in the visualization stage. This will improve the situation with the upgrading of the skills of pilots.

\section{REFERENCES}

[1] A. A. Krasovskiy, E. V. Lapshin, and N. K. Yurkov, Matematicheskoye modelirovaniye dinamiki poleta letatel'nogo apparata, Penza: Penza State University, 2008, p. 259.

[2] http://www.cae.com/about-cae/media-centre/photogallery/?LangType=1033 (date of the application 03.05.2016).

[3] V. R. Roganov, M. J. Miheev, A. N. Seredkin, V. O. Filippenko, and A. V. Semochkin, "Capacity assessment of visual conditions imitators," Eastern European Scientific Journal, No 6, pp. 321-326, 2014.

[4] S. Kozlov, N. Elykov, and I. Belago. "Visualization of rain effects for driving simulators," GraphiCon'2006 - International Conference on Computer Graphics and Vision Proceedings, 2006, pp. 363-366.

[5] S. I. Vyatkin, B. S. Dolgovesov, and A. V. Yesin, "Visualization of 3d clouds using free forms," Computer Graphics \& Geometry, vol. 4, No 2, pp. 25-49, 2002

[6] N. K. Yurkov, A. N. Andreev, A. M. Danilov, B. V. Klyuev, É. V. Lapshin, and A. V. Blinov, "Information models for designing conceptual broad-profile flight simulators," Measurement Techniques. N.Y., Springer, vol. 43, No. 8, pp. 667-672, August 2000.

[7] V. R. Roganov, E. A. Asmolova, A. N .Seredkin, M. V. Chetvergova, N. B. Andreeva, and V. O. Filippenko, "Problem of virtual space modelling in aviation simulators," Life Science Journal, vol. 11, pp. 1097, 2014.

[8] S. I. Vyatkin, B. S. Dolgovesov, and N. R. Kaipov, "Texture mapping on plane and curvilinear surfaces, free shapes and volumes," Autometry, No. 1, pp. 17-24, 2002.

[9] https://yandex.ru/images/search?img_url=http\%3A\%2F\%2Fhealthfitnes.ru\%2Fcimg\%2F2015\%2F041112\%2F2702735\&text=авиационн ые\%20тренажёры\&noreask=1\&pos=2\&lr=49\&rpt=simage $($ date of the application 03.05.2016).

[10] https://yandex.ru/images/search?img_url=http\%3A\%2F\%2Fi.ytimg.com $\%$ 2Fvi\%2FZAr0gBQKsbI\%2Fhqdefault.jpg $\& \mathrm{p}=14 \& \mathrm{text}=$ вид $\% 20$ на $\% 2$ 0экране \%20радиолокатора\&noreask $=1 \&$ pos $=562 \& \mathrm{rpt}=$ simage $\& \mathrm{lr}=49$ (date of the application 03.05.2016).

[11] https://yandex.ru/images/search?text=вид\%20на\%20экране\%20радиол окатора\&stype $=$ image $\& 1 \mathrm{r}=49 \&$ noreask $=1 \&$ parentreqid=1489040829514388-8851998735373127172225942-ws11$383 \&$ source $=$ wiz (date of the application 03.05.2016).

[12] https://yandex.ru/images/search?img_url=http\%3A\%2F\%2Fhealth fitnes.ru\%2Fcimg\%2F2015\%2F041121\%2F0007439\&p=2\&text=пкбм $\% 20$ пенза\&noreask $=1 \&$ pos $=101 \& \mathrm{rpt}=$ simage $\& \mathrm{lr}=49$ (date of the application 03.05.2016).

[13] https://yandex.ru/images/search?img_url=http\%3A\%2F\%2Fru.transas.c om $\% 2$ FMedia\%2FDefault $\% 2$ FImageGalleries $\% 2$ FFNPTMi171_Images\%2F690_i_1.png\&text=авиационные\%20тренажёры\%20к артинки\&noreask $=1 \&$ pos $=34 \& 1 \mathrm{r}=49 \& \mathrm{rpt}=\operatorname{simage}$ (date of the application 03.05.2016). 